\title{
Delayed Dissociation Spectra of Survivor Ions from High-Energy Collisional Activation*
}

\author{
P. Thibault, A. J. Alexander, and R. K. Boyd \\ Institute for Marine Biosciences, National Research Council, Halifax, Nova Scotia, Canada

\section{K. B. Tomer} \\ Laboratory of Molecular Biophysics, National Institute of Environmental Health Sciences, \\ Research Triangle Park, North Carolina, USA
}

\begin{abstract}
Collisional activation (CA) of large ions at kiloelectronvolt energies is accompanied by unexpectedly large losses of translational energy, which vary with the nature of the collision gas. Previous investigations have concentrated upon subsequent fragmentations occurring within a time window covering a few $\mu$ s immediately following collision, using massanalyzed ion kinctic energy spectrometry. In the present work, survivor ions were selected for specified values of translational energy loss, and their internal energy contents assessed via their subsequent unimolecular fragmentation reactions within a later time window. Beam collimation was also applied when circumstances permitted to impose angular selection, thus minimizing cross talk between effects of collisional scattering and energy dispersion. It was shown that internal excitation of the reactant ion can account for only a small fraction of the observed loss of translational energy. The recoil energy of the target is thus the principal sink for the translational energy loss, since the latter was always chosen to be less than the lowest excitation energy of the target. This conclusion is shown to be consistent with theoretical models of the CA process. The practical implications of these conclusions for CA of large ions at kiloelectronvolt energies are discussed. ( $\mathrm{f}$ Am Soc Mass Spectram 1993, 4, 845-854)
\end{abstract}

I $\mathbf{t}$ is generally accepted [1, 2] that the collisioninduced dissociation (CID) of ions may meaningfully be divided into two well-separated processes, that is, a fast collisional activation (CA) step followed by the dissociation itself on a slower time scale. The present experimental work deliberately separates the CA step from the subsequent dissociation by subjecting mass-selected reactant ions to $C A$, then selecting for scattering angle and/or translational energy loss, and finally monitoring their delayed unimolecular fragmentation reactions. If some feature of the fragment ion spectrum has been independently calibrated in terms of internal energy, such experiments in principle permit some insight into details of the CA process.

Derrick and co-workers [3-6] were the first to draw attention to an unexpected feature of CID of large organic ions at kiloelectronvolt energies. It was found, when these CID reactions were studied using massanalyzed ion kinetic energy spectrometry (MIKES), that

\footnotetext{
*NRCC No. 34882.

${ }^{\dagger}$ Current address: Bristol-Myers-Squibb Pharmaceutical Research Institute, P.O. Box 4755, Syracuse, NY 13221.

Address Ieprint requests to Robert $K$. Boyd Institute for Marine Biosciences, National Reseanch Council, 1411 Oxford Street. Halifax, Nova Scotia, B3H 37.1, Canada.
}

the fragment ions appeared to have been formed from reactant ions that had undergone surprisingly large losses of translational energy, $\Delta \mathrm{T}_{\mathrm{R}}$. Conversion of translational to internal energy is of course the basis for the CID method, but it was the surprisingly large magnitude of these energy deficits (up to $50 \mathrm{eV}$ in some cases) that required some explanation. Similar observations by other workers [7-9] confirmed the general validity of the original findings [3-6].

The most obvious interpretation of such observa tions is that most of $\Delta \mathrm{T}_{\mathrm{K}}$ is transformed into $\epsilon_{\mathrm{K}}$, the internal energy of the reactant ion, to meet large kinetic shift requirements. As emphasized in the companion article [10], kinetic shift requirements cannot casily explain crucial features of the dependence of $\Delta T_{R}$ on the nature of the collision gas for a fixed population of reactant ions and a fixed experimental time window for observation of the CID products. Thus, large values of $\Delta \mathrm{T}_{\mathrm{R}}$ are observed for very light collision targets $\left(\mathrm{H}_{2}, \mathrm{D}_{2}, \mathrm{He}\right)$, while much smaller values of $\Delta T_{R}$ are obtained for all others (Ne, $A r, K r$, $\mathrm{Xe}, \mathrm{N}_{2}$ ). The peak shapes observed in CID-MIKES experiments are also strongly dependent on the nature of the target gas. These target-dependent observations are difficult to understand on the basis of the kinetic shift effect alone. 
Two different explanations of this discrepancy have been proposed. That suggested by Bricker and Russell [7] proposes that those $C A$ collisions, which lead to observable CID products, are necessarily accompanied by excitation (possibly including ionization) of the target. Derrick and co-workers [3-6] and ourselves [9, 10] have proposed that recoil translational energy of the target is the major sink for $\Delta \mathrm{T}_{\mathrm{R}}$. In the preceding article [10], this question was approached by investigating the angle dependence of translational energy spectra of survivor ions from the CA process. The same general trends were observed [10] as in previous investigations [3-9] of fragment ions by CID-MLKES, even though the great majority of the data were obtaired for values of $\Delta T_{R}$ that were less than the lowest excitation energies of the inert gas targets. Moreover, the data obtained using deuterium as the collision target were barely distinguishable from those for helium, and these results for $\mathrm{He}$ and $\mathrm{D}_{2}$ were well separated from those for all other target gases. Both of these experimental features [10] present serious problems for the interpretation in terms of target excitation [7] but are consistent with theories of inelastic scattering $[11,12]$, which assign the major sink for $\Delta T_{R}$ to be $\mathrm{T}_{\mathrm{G}}$, the recoil energy of the collision gas target.

The experimental approach used here involves an extension of previous work by Cerny and co-workers $[13,14]$, who studied the low-energy tails on the kinetic energy peaks corresponding to surviving reactant ions studied by CID-MIKES. The ions contributing to these tails must have suffered considerable translational energy losses in the CA step but had not dissociated during the flight from the collision cell to the exit plane of the analyzer. These collisionally activated ions are exploited here by selecting activated but intact reactant ions and characterizing their internal energy contents via their subsequent (delayed) unimolecular dissociations. Although the use of such a "chemical thermometer" for measuring internal energies of ions involves intrinsic uncertainties, there is presently no realistic alternative (e.g., spectroscopy) for ions of sufficient complexity to be of chemical interest in the present context. Preliminary accounts of parts of this work have been published previously [15, 16]. It is worthwhile to note that the energy tails exploited here are different from those investigated recently by Ballard and Gaskell [17], who clarified the origins of the extensive tailing signals in MIKE spectra of precursur ions produced by fast-atom bombardment (FAB) ionization using liquid matrices. The energy tails $[13,14]$ of interest here are much less extensive, disappear when no collision gas is present, and also are observed for precursor ions formed by all ionization techniques.

Experiments in which some control over the internal energies of reactant ions was exerted via selection of their translational energies were previously reported by Proctor et al. [18, 19]. In these experiments, the reactant ions were themselves formed by unimolecular dissociations of their precursor ions in a field-free region. Because the width of a metastable peak is a direct reflection of that portion of the reactant's internal energy released as relative translational energy $W$ of the fragments, experimental selection of $W$ will, for a fixed initial value $\epsilon_{\mathbb{R}}^{\circ}$, also specify the combined internal energy of the dissociation products. This work $[18,19]$ emphasized the importance of collimation of the ion beam in such experiments. The same simple principle of energy conservation also underlies the present work, except that now the translational energy loss $\Delta \mathrm{T}_{\mathrm{R}}\left(=\mathrm{T}_{\mathrm{R}}^{\circ}-\mathrm{T}_{\mathrm{R}}\right)$ of the reactant ion plays the role of $W$ in the previous work $[18,19]$ :

$$
\begin{aligned}
\Delta \mathrm{T}_{\mathrm{R}} & =\left[\left(\epsilon_{\mathrm{R}}-\epsilon_{\mathrm{R}}^{\mathrm{o}}\right)+\left(\epsilon_{\mathrm{G}}-\epsilon_{\mathrm{G}}^{\mathbf{o}}\right)+\mathrm{h} \nu\right]+\Delta \mathrm{T}_{\mathrm{G}} \\
& =\mathrm{Q}+\Delta \mathrm{T}_{\mathrm{G}}
\end{aligned}
$$

where superscript ${ }^{\circ}$ denotes a precollision value, $\Delta \mathrm{T}_{\mathrm{G}}$ $\left(-T_{G}-T_{G}^{o}\right)$ the translational energy gained by $G$ in the collision, and $Q$ the total inelasticity. As discussed in the preceding article [10], it is highly improbable that radiation cooling could provide a significant sink for $\Delta \mathrm{T}_{\mathrm{R}}$. By choosing inert gas targets $\mathrm{G}$, target excitation $\left(\epsilon_{\mathrm{G}}-\epsilon_{\mathrm{G}}^{\mathrm{P}}\right)$ can be removed from consideration by restricting experimental values of $Q$ to be less than the appropriate first excitation energies. If the values of $\left(\epsilon_{R}-\epsilon_{R}^{0}\right)$ can be estimated independently (e.g., by measuring intensity ratios of fragment ions for some reactant ion for which such a ratio has been previously calibrated in terms of $\epsilon_{\mathbf{R}}$ ), eq 1 permits estimates of $\Delta T_{G}$ to be made from measurements of $\Delta T_{R}$. All quantities in eq 1 , other than $\epsilon_{\mathrm{G}}$, are to be understood as most-probable values representative of appropriate distributions.

A major problem in the present work was to find suitable precursor ions. The fragmentation characteristics of the molecular radical cation $\mathrm{C}_{10} \mathrm{H}_{14}^{+}$of butylbenzene have been extensively investigated [20-26] as a function of $\epsilon_{R}$ and preliminary experiments [15] demonstrated that an energy tail of adequate intensity could be produced in CA experiments. However, this ion is still fairly small in the context of the published work [3-9] on large values of $\Delta T_{R}$. Unfortunately, no such information of the desired quality is available for larger ions (typically protonated peptides) of interest in this context, and it was necessary to adopt a more empirical approach described at appropriate points in the following discussion. An additional problem in the present work was that of limited reactant beam intensity. Although exceptions do exist, it seems to be a general rule that beam intensities of protonated peptides produced by FAB decrease significantly with increasing molecular weight. Because both beam collimation and selection of reactant ions from the lowenergy tail imply severe losses in reactant intensity, it is essential to use reactant ions available in reasonable FAB yields. For this reason, the reactant systems studied here are not truly large in the sense intended by Derrick and co-workers [3-6]. 
It is appropriate to acknowledge here the importance to the present work of the early studies of angleresolved mass spectrometry by Cooks and co-workers [27-35], whose work established the importance of the angular dependence of $\mathrm{CA}$ of organic ions in mass spectrometers and demonstrated its analytical potential. However, it is difficult to extract meaningful information about the CA step from most of these carly experiments, principally because the experimental techniques used resulted in convolution of the effects of angular scattering of the reactant ion with angular distributions of the fragments due to the kinetic energy release associated with the dissociation $[36,37]$. One approach to this difficulty involves coincident detection of ionic and neutral fragments [38, 39] in specialized apparatus incorporating a position-sensitive detector. This elegant technique is, however, limited in its range of applications. The present work avoids the problem of convoluting collisional scattering of reactant ions with angular distributions of fragments due to kinetic energy release by scparating the two steps from one another, as described below.

\section{Experimental}

Experiments were conducted using a VG Analytical ZAB-EQ tandem hybrid instrument (BEqQ configuration) and a VG Analytical $\mathrm{ZAB}-4 \mathrm{~F}$ tandem doublefocusing (four-sector) instrument $\left(B_{1} E_{1} E_{2} B_{2}\right.$ configuration), each equipped with a VG $250 \mathrm{~J}$ datasystem. In collision experiments, the collision gas was used at as low a pressure as possible consistent with adequate signal/noise ratios in the resulting spectra. These pressures were such that reactant beam intensities were attenuated by 15-20\%; under these conditions, the most probable outcome (apart from no collisions at all) was a single-collision event. Only some $10 \%$ of the total beam attenuation was attributable to ion loss due to multiple collisions.

The $\mathrm{BEqQ}$ instrument used here has all the same features as those described previously [40] for a related instrument but, in addition, has a high-field magnet with extended mass-range optics and a VG 11-250 datasystem for instrument control and data acquisition. The facilities for beam collimation and angular selection have been described [10]. Unfortunately, no such facilities were available on the four-sector instrument used in the present work. The present experiments used the first $\mathrm{BE}$ double-focusing analyzer in MIKES mode (i.e., use of $B$ [or $B_{1}$ ] to mass select the reactant ion and of $E$ [or $E_{1}$ ] to select the translationa] energy of the ions transmitted). In the present work, the ions selected for transmission to the second mass analyzer were reactant ions with translational energies less than that observed in the absence of collision gas. Delayed unimolecular dissociations of these selected reactant ions were then studied either in the field-free region between $E_{1}$ and $E_{2}$ (four-sector instrument) or within the RF-only quadrupole $\mathrm{q}$ (hybrid instrument).
The electron ionization sources were uperated at 200 ${ }^{\circ} \mathrm{C}$, with a nominal electron energy of $70 \mathrm{eV}$ and a trap current of $100 \mu \mathrm{A}$. In the case of reactant ions produced by FAB ionization, the primary beam used with the hybrid instrument was a $30 \mathrm{keV} \mathrm{Cs}^{+}$beam. The liquid matrix used was acidified glycerol or thioglycerol. Further experimental details are best left until each experiment is discussed in turn.

The butylbenzene was obtained from Aldrich Chemical Co., Milwaukee, WI, and the Leu-enkephalin from Sigma Chemical Co., St. Louis, MO; both were used as received. The galactose was also obtained from Sigma and was converted to its penta- $\mathrm{d}_{3}$-acetyl derivative by reaction with perdeutero-acetic anhydride.

\section{Results and Discussion}

To assess the internal energies of reactant ions, it is necessary to choose a system for which some feature of its fragment spectrum, typically an intensity ratio of two fragment ions, has been calibrated against internal energy. Indeed, the only realistic alternative is to monitor variations in the kinetic energy release upon fragmentation [41]. Methods based upon optical spectroscopy are likely to be useful only for ions that possess a high degree of molecular symmetry.

Currently, the best known of these calibrated systems involves the intensity ratio for product ions at $\mathrm{m} / z 92$ and 91 , formed from the molecular radical cation of butylbenzene [20-26]. Successive expulsions of $\mathrm{CO}$ from $\mathrm{W}(\mathrm{CO})_{6}^{+}$have also been calibrated in terms of internal energy [42-44]. Although neither of these is a large ion in the present context fonly 66 and 33 vibrational modes, respectively), it was decided to include one of them in the present study as a relatively well-understood test case. Butylbenzene was chosen because of the larger number of vibrational modes and also because the closely similar masses of the two fragment ions would minimize any mass discrimination effects. In the case of larger ions, more characteristic of the biological molecules of interest, no case is known to us for which any kind of calibration of fragmentation characteristics in terms of internal energy is available. Accordingly, the best that could be achieved for such systems was to establish an empirical index for internal energy rather than an absolute calibration. However, as explained below, it was possible to define these empirical indices so as to provide upper limits to the internal energy $\epsilon_{\mathrm{R}}$, and this feature was important for the main conclusions of the present work.

\section{Butyibenzene Molecular Ions}

Preliminary experiments [15] established that, under CA-MIKES conditions using helium, the survivor ions showed an energy spectrum with an apprcciable lowenergy tail. Use of the RF-only quadrupole cell of the 
$\mathrm{BEqQ}$ hybrid instrument, as the second (unimolecular) reaction region, showed that the higher-energy channel $(m / z$ 91) was not accessible to any significant extent. Therefore, at values of $\epsilon_{R}$ for which $k_{92}$ (the rate constant for formation of $m / z$ 92) falls in the range $10^{4}-10^{5} \mathrm{~s}^{-1}$, to match the experimental reaction time window for the quadrupole cell, the ratio $k_{91} / k_{92}$ must be less than about $10^{-2}$ (as defined by the signal/noise ratio of the observed spectra). This condition is fulfilled by both of the rival calibrations $[23,25]$ discussed below (see Figure 2 later). To access a more appropriate reaction time window following $C A$, the field-free region between $E_{1}$ and $E_{2}$ of the $B_{1} E_{1} E_{2} B_{2}$ instrument was used as the reaction region to probe the unimolecular reactions of the survivor ions. Unfortunately, this instrument was not equipped with additional slits and restrictors, nor with a moveable $z$-aperture, to permit angular selection.

The intensity ratio for the fragments at $m / z 91$ and 92 was first studied as a function of $\epsilon_{R}$ by laser photodissociation of a fast beam of $\mathrm{C}_{10} \mathrm{H}_{14}^{+}$ions, formed by $70 \mathrm{eV}$ electron ionization of butylbenzene at different ion source temperatures $[20,21]$; this work clearly demonstrated the importance of preexcitation energy $\epsilon_{\mathrm{R}}^{\circ}$ in this system. Subsequent work used chargeexchange ionization to vary the internal energies of $\mathrm{C}_{10} \mathrm{H}_{14}^{+}$ions observed to fragment within a chemical ionization source [22]. Laser photodissociation of cold $\mathrm{C}_{10} \mathrm{H}_{14}^{+}$ions, formed by $11 \mathrm{eV}$ electron ionization and allowed to cool radiatively in an ion cyclotron resonance (ICR) cell, has also been employed [23]. An attempt [24] to rationalize the differences among these data invoked differences in the experimental techniques, including variations in reaction time windows and in preexcitation internal energies. More recently [25], the photoelectron-photoion coincidence (PEPICO) technique was applied to the butylbenzene problem. The most definitive results are those obtained in the ICR photolysis [23] and PEPICO [25] experiments.

In the present experiments, the $\mathrm{C}_{10} \mathrm{H}_{14}^{+}$ions were formed by $70 \mathrm{eV}$ electron ionization at $200^{\circ} \mathrm{C}$, accelerated to $8 \mathrm{keV}$, and transmitted to the collision cell (between $B_{1}$ and $E_{1}$ ) containing helium. No angular selection was possible, so the results correspond to an integration over $\theta_{R}$, the laboratory-frame scattering angle of the reactant ion. In the absence of collision gas, the energy width of the reactant beam was $3 \mathrm{eV}$ (full width at $50 \%$ of peak height), so values of $\Delta T_{R}$ of $0,3,6,9$, and $12 \mathrm{eV}$ were selected in turn. and the corresponding ions transmitted to the field-free region between $\mathrm{F}_{1}$ and $\mathrm{E}_{2}$. The unimolecular reaction products at $m / z 91$ and 92 , formed in this third field-free region after $\mathrm{CA}$ in the second field-free region, were recorded via a linked scan at constant $E_{2} / B_{2}$. The reaction time window can be estimated to be $0-6 \mu$ s after energy selection at the energy-resolving slit (image slit of the electric sector), corresponding to 9-15 $\mu$ s after collision, which in turn was some $10 \mu \mathrm{s}$ after ionization. The results obtained are summarized in Figure 1. Note that since the lowest excitation energy of helium is $19.8 \mathrm{eV}$, values of $\Delta \mathrm{T}_{\mathrm{R}}$ up to $12 \mathrm{eV}$ cannot be accounted for on the basis of target excitation [i.e., $\left(\epsilon_{G}-\epsilon_{G}^{g}\right)$ in eq 2 is zero].

The most recent calibrations $[23,25]$ of the intensity ratio for the ions at $m / z 91$ and 92 , in terms of internal energy $\epsilon_{R}$, were expressed as fragmentation rate constants $k\left(\epsilon_{\mathrm{R}}\right)$ for each of the two channels, using RRKM unimolecular rate theory with very similar values for the subsidiary molecular parameters. These results have been extracted from the graphs in the original studies [23, 25], inevitably with some reading errors (a few percent), and are reproduced in Figure 2 . The two sets of curves agree as to the value of $\epsilon_{R}(4.0 \pm 0.1 \mathrm{eV})$ at which the crossover occurs. However, the rate constants deduced from the ICR/photolysis experiments [23] are lower than those from the PEPICO experiments [25] by factors in the range of 20-30. It does not seem possible to understand the reasons for this discrepancy on the basis of the published information [23, 25].

Although the present experiments are certainly not sufficiently discriminating to choose between the competing sets of curves, it does appear that a more self-consistent interpretation of the present results is possible in terms of the $\mathrm{k}\left(\epsilon_{\mathrm{R}}\right)$ values deduced from the ICR/photolysis experiments [23]. Thus, if the simplest possible assumption is made, that is, that the observed intensity ratio in the present work corresponds directly to the ratio of the average rate constants, then differences between $\log \left(k_{92}\right)$ and $\log \left(k_{91}\right)$ in the range 1.1 to

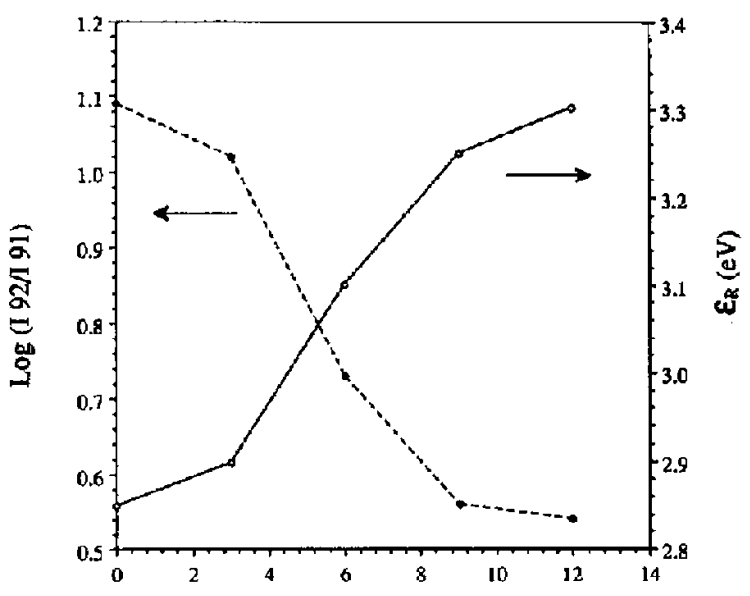

$\Delta T_{R}(\mathrm{eV})$

Figure 1. Results for unimolecular fragmentations of butylbenzene molecular ions following collisional activation (helium, 8 $\mathrm{keV})$ but within a time window 9-15 $\mu$ s after collision. The reactant ions were selected via their translational energy losses $\Delta T_{R}$, with integration over the full range of $\theta_{R} .=$ Logarithm of ratio of intensity for $m / z 92$ to that of $m / z 91 ; 0=$ Average internal energy $\epsilon_{R}$ of butylbenzene molecular ions, as deduced from the intensity ratio interpreted via the rate constant curves of Chen et al. [23], see Figure 2. 


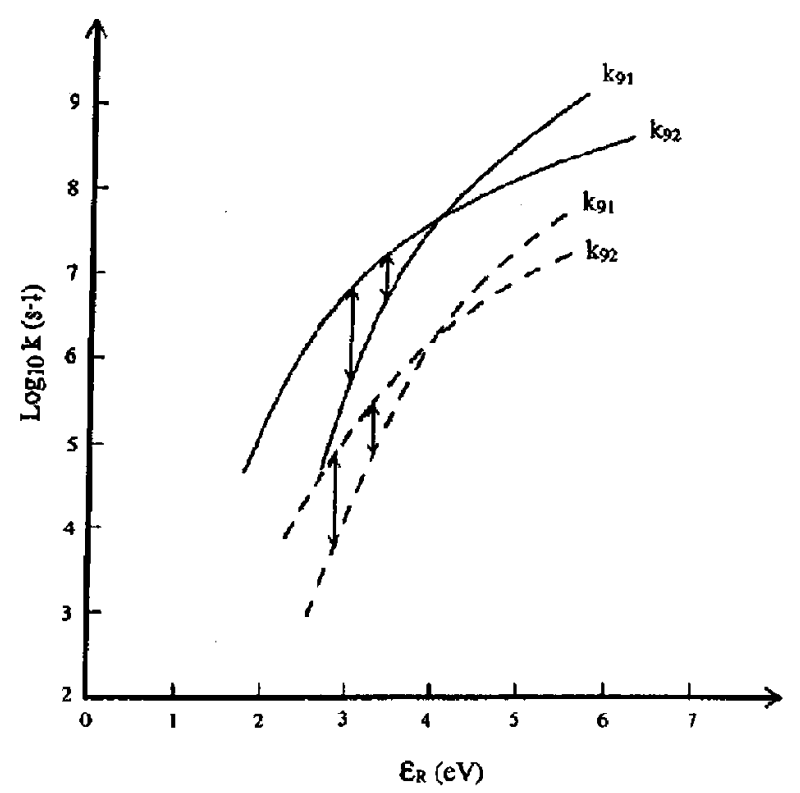

Figure 2 Unimolecular rate constants for butylbenzene molec1lar ions fragmenting to $m / z 91$ and 92 , as a function of internal energy $\epsilon_{R},-=$ adapted from work of Baer et al. [25], PEPICO data; $\ldots . .-$ adapted from work of Chen et al. [23], ICR/photolysis data. The vertical double-arrows represent ratios of rate constants (i.e., differences between logarithms) equal to the largest and smallest experimental intensity ratios for $m / z \quad 92$ and 91 , observed in Figure 1.

0.55 (Figure 1) must be sought; these are shown as the vertical arrows in Figure 2, and the corresponding ranges of $\epsilon_{R}$ are not too different for the two sets of rate-constant curves. However, the experimental time window, fixed by the instrumental parameters, was 9-15 $\mu$ s after collision so that the absolute values of the rate constants must have been of the order of $10^{5}$ $s^{-} 1$. This requirement of any interpretation of the present experiments is reasonably satisfied by the ICR/photolysis rate constants [23], while those from the PEPICO experiments [25] are too high by an order of magnitude (Figure 2) if the ratio $\mathrm{k}_{91} / \mathrm{k}_{92}$ is simultaneously required to fall in the correct range. For this reason, the values of $\epsilon_{R}$ plotted in Figure 1 are those deduced from the lower set of rate constants [23], although the PEPICO calibration [25] would give $\epsilon_{\mathrm{R}}$ values higher by only $0.2 \mathrm{eV}$.

The most striking result evident in Figure 1 is the difference between the selected values of $\Delta T_{R}(0-12$ $\mathrm{eV})$ and the corresponding values of $\epsilon_{\mathrm{R}}$; the latter vary between $2.85 \mathrm{eV}$ (at $\Delta \mathrm{T}_{\mathrm{R}}=0$ ) and $3.30 \mathrm{eV}$ (at $\Delta \mathrm{T}_{\mathrm{R}}=12$ $\mathrm{eV}$. The reactant ions reaching the collision cell may be described in terms of a distribution of $\epsilon_{\mathrm{R}}^{\circ}$ ranging from (presumably) zero to values high enough to account for the intense metastable peak for production of $m / z$ 92. Note that the observation (Figure 1) of $m / z$ 91 fragments under CID conditions at $\Delta \mathrm{T}_{\mathrm{K}}=0$ (i.e, at nominally zero energy deposition), is inconsistent with the absence of $m / z$ from the metastable ion spec- trum. This discrepancy undoubtedly reflects the cross talk between collisional scattering and energy dispersion in the absence of any angular collimation facilities on the four-sector instrument. Nonetheless, since the critical energies for production of $m / z 92$ and $m / z 91$ are $0.99-1.1 \mathrm{eV}$ and $1.61 \mathrm{eV}$, respectively $[23,25]$, the values of $\epsilon_{\mathrm{R}}^{\circ}$ must extend from zero to $1.1-1.6 \mathrm{eV}$, and possibly (Figure 1) as high as $2.85 \mathrm{eV}$. On the other hand, the postcollision values of $\epsilon_{R}$ (Figure 1) extend up to only $3.3 \mathrm{eV}$. Therefore, the maximum possible mean value for $\left(\epsilon_{R}-\epsilon_{R}^{0}\right)$ is $3.3 \mathrm{eV}$, and this refers to collisions for which $\Delta T_{R}$ is $12 \mathrm{eV}$. If the possibility of radiative cooling of these activated ions is discounted [10], one is forced to the conclusion that a maximum of $25 \%$ of $\Delta \mathrm{T}_{\mathrm{K}}$, and possibly considerably less, was deposited as internal energy in the $\mathrm{C}_{10} \mathrm{H}_{14}^{+}$reactant ions. The only other available sink for $\Delta T_{R}$ is the recoil energy of the helium target, since the lowest excitation energy for the latter is $19.8 \mathrm{eV}$.

It is possible to relate these experiments to the collision theories [11, 12] described in the companion article [10]. Figure 3 shows results of calculations based on the Uggerud-Derrick model [12] for $8 \mathrm{keV} \mathrm{C}{ }_{10} \mathrm{H}_{14}^{+}$ ions colliding with helium. Values of $\Delta T_{R}$ are shown for values of $\mathrm{m}_{\mathrm{a}}=12 \mathrm{Da}$ (helium interaction with a single carbon atom) and $77 \mathrm{Da}$ (interaction with the phenyl ring); $\Delta \mathrm{T}_{\mathrm{F}}$ values calculated for $\mathrm{m}_{\mathrm{a}}=57 \mathrm{Da}$ (interaction with the alkyl side chain) were close to the $77 \mathrm{Da}$ values. The maximum value of $\theta_{\mathrm{R}}$ in the apparatus used was $0.29^{\circ}$, which corresponds (Figure 3 ) to calculated $\Delta T_{R}$ values in the range $7-9 \mathrm{eV}$. The same calculations also yield values of the ratio $\left(Q / \Delta \mathrm{T}_{R}\right), 0.23$

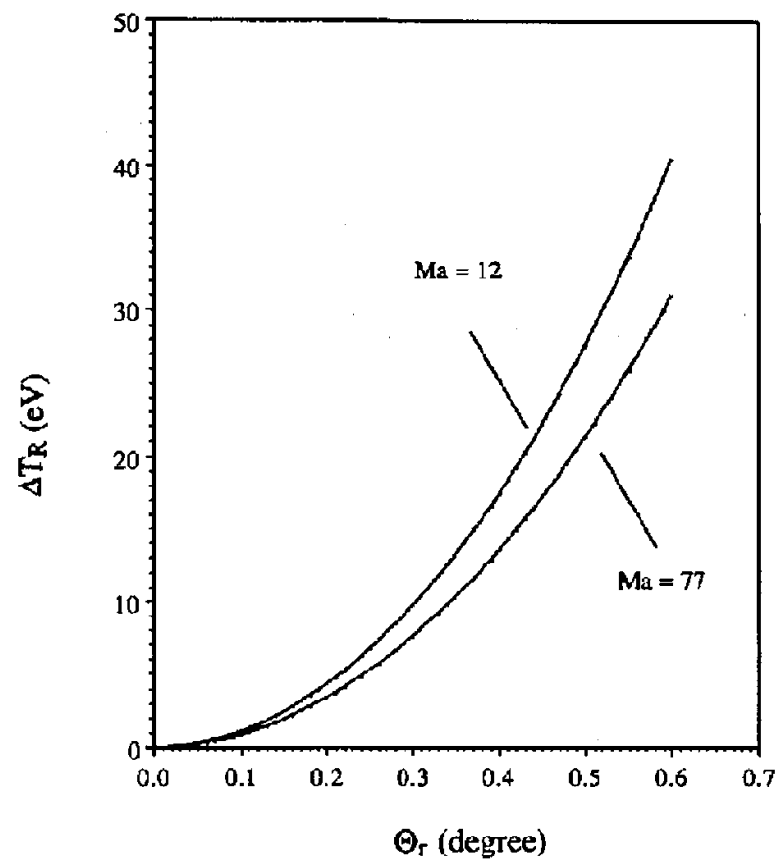

Figure 3. Results of calculations based on the Uggerud-Derrick collision model [12], for $8 \mathrm{keV} \mathrm{C}_{10} \mathrm{H}_{1 i}^{+}$ions colliding with helium. $X \mathrm{~m}_{\mathrm{a}}=12 \mathrm{Da} ; \mathrm{O} \mathrm{m}_{\mathrm{s}}=43 \mathrm{Da}$. 
for $\mathrm{m}_{\mathrm{a}}=12$ and 0.059 for $\mathrm{m}_{\mathrm{a}}=43$. Thus, maximum values of $Q$, equivalent to $\left(\epsilon_{R}-\epsilon_{R}^{o}\right)$ in this case, are predicted to be in the range $0.42-2.1 \mathrm{eV}$, where the latter value corresponds to $\Delta \mathrm{T}_{\mathrm{R}}=9 \mathrm{eV}$. These predictions, based upon the Uggerud-Derrick model [12] are in remarkable agreement with the conclusions reached above from consideration of the experimental data (Figure 1). The closeness of this quantitative agreement may be fortuitous, but it does lend support to the main conclusion drawn from the experimental results, that is, only a small fraction of $\Delta T_{R}$ can be accounted for by the inelasticity. It would have been preferable to repeat the $\mathrm{C}_{10} \mathrm{H}_{14}^{+}$experiments with a heavier target, but the low-energy tails in the MIKE spectra for the survivor ions were too limited to permit this.

\section{Leu-Enkephalin}

The fragmentation of the protonated form of this simple pentapeptide (YGGFL-OH), in the RF-only quadrupole collision cell of the hybrid instrument used in the present work, has been studied extensively [45]. The intensity ratio for the $b_{3}$ and $y_{2}$ " fragments (at $m / z 278$ and 279 , respectively) was shown [45] to be a converient index of the degree of internal excitation with no possibility of mass discrimination effects. However, use of this intensity ratio as an empirical index of internal energy content in the present experiments gave disappointing results, in that only a limited range of ratios was observed. It was found to be more fruitful to employ the intensity ratio for the $b_{4}$ and $a_{4}$ fragments $(m / z 425$ and 397 , respectively), since a wider range of intensity ratios could be accessed (more sensitive to internal energy) at the expense of a greater risk of mass discrimination.

No absolutc calibration, such as that available [23, 25] for the butylbenzene system, could be obtained for Leu-enkephalin. Instead, the intensity ratio for the chosen fragment ions was studied under low-energy CID conditions corresponding to predominantly single collisions (15\% attenuation of precursor intensity). This series of experiments was conducted in the RF-only quadrupole collision cell of the same BEqQ mass spectrometer, under tuning conditions identical to those used subsequently to investigate the unimolecular dissociations of $R^{+}$ions selected via $\Delta T_{R}$ values. The experimentally determined ratios, for both argon and xenon as collision gases, are shown in Figure 4 as a function of the center-of-mass collision energy $\mathrm{T}_{\mathrm{COM}}$ (equivalent to the maximum inelasticity $Q_{\max }$, see eq 8 of ref 10 . The values of this particular intensity ratio for the two collision gases agree very well on this basis. This is not an absolute calibration in any sense, since the distribution function for $Q$ (between the limits zero and $T_{C O M}=Q_{\max }$ ), under the conditions used to obtain the data in Pigure 4 , is wholly unknown. However, the agreement between the data obtained using argon and xenon, the linearity of the

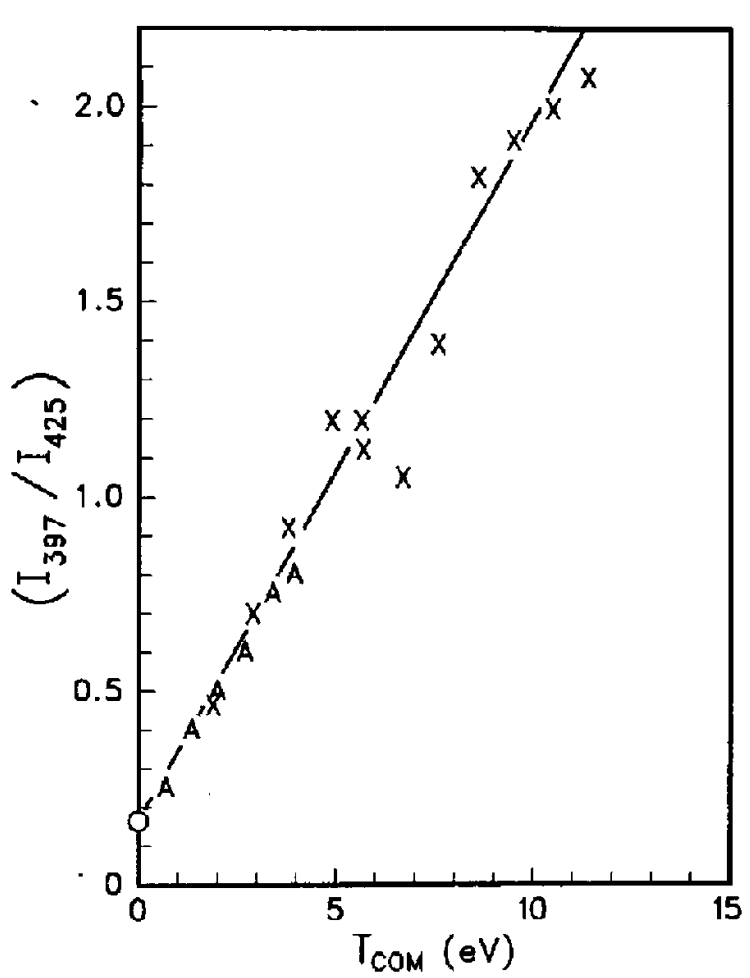

Figure 4. Intensity ratio for $b_{4}$ and $a_{4}$ ions $(m / z 425$ and 397 , respectively) from CID of $(M+H)^{+}$ions of Leu-enkephalin $(\mathrm{m} / z$ 556) in RF-anly collision cell $\mathrm{q}$ of a $\mathrm{BEqQ}$ hybrid instrument. Single-collision conditions (15-20\% collisional attenuation of reactant-beam intensity). The elastic limit center-of-mass collision energy $T_{C O M}$ represents $Q_{M A X}$, the maximum inelasticity available. The actual mean value of $Q$ was taken to be a fraction $f$ of $Q_{\text {MAX }} . X=$ Xenon collision gas; $A=$ Argon collision gas; $\mathbf{O}=$ Intensity ratio measured without collision gas.

combined data, and the fact that the intensity ratio intercept derived by extrapolation to $\mathrm{T}_{\mathrm{COM}}=0$ agrees closely (Figure 4) with the value obtained in the absence of collision gas (both experiments should reflect the effect of $\epsilon_{\mathrm{R}}^{\mathrm{O}}$ only), all suggest that the mean value of $Q$ in these low-energy CID experiments was some (unknown) fixed fraction $f$ of $T_{\text {COM }}\left(=Q_{\max }\right)$. It is this result that was used as the basis for this intensity ratio as an empirical index of internal energy.

Typical results for delayed dissociations of ions selected for $\Delta T_{k}$, following $C A$ with helium at $8 \mathrm{keV}$, are shown in Figure 5. The intensity of the reactant beam was not sufficient to permit beam collimation and angular selection, so these results represent integrations over $\theta_{\mathrm{R}}$. The hybrid instrument has a disadvantage in experiments of this kind in that the internal energy of the ions is probed only after a time delay, corresponding to the flight time from the energy resolving slit to the RF-only quadrupole. Thus, the reactant ions actually probed in the hybrid instrument must have been preferentially depleted of the population at higher $\varepsilon_{\mathrm{R}}$ values due to dissociations while in flight. (For ions of $m / z 556$, initially at $8 \mathrm{keV}$ and 


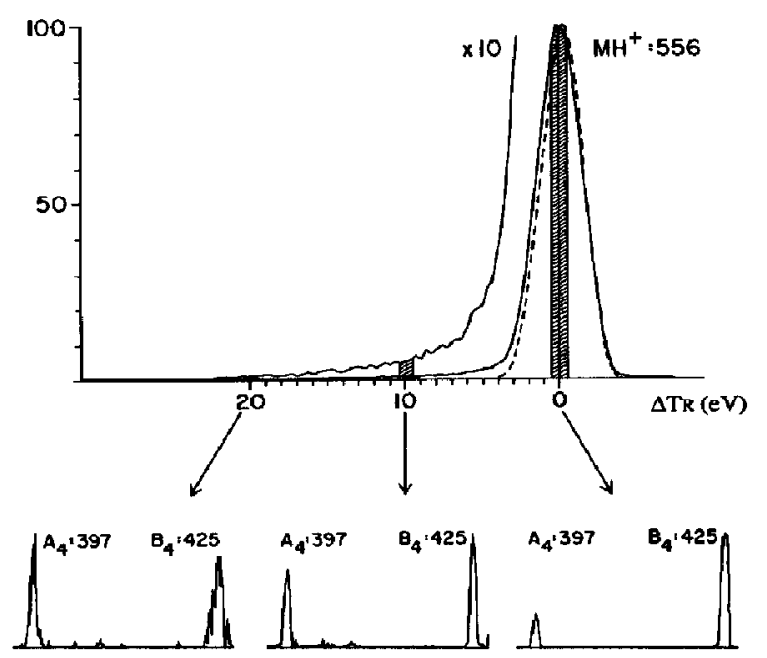

Figure 5. Delayed dissociations of $(M+H)^{+}$ions of Leu-enkephalin ( $m / z 556$ ), following collisional activation (helium, 8 $\mathrm{keV})$ and selection for translational energy loss $\Delta T_{R}$. No angular collimation was applied. Dissociations were observed in the RF-arly cell (q) of a BEqQ hybrid instrument with no collision gas and pumped to an indicated pressure of $10^{-8}$ mbar. The peak shape drawn as a dashed curve corresponds to that obtained in the absence of collision gas.

decelerated to $30 \mathrm{eV}$ within $q$, the flight time from energy resolving slit through deceleration to $q$ in the $B E q Q$ apparatus was estimated to be $15 \mu \mathrm{s}$, while residence time within $\mathrm{q}$ was a further $40 \mu \mathrm{s}$.) The experimental data are plotted in Figure 6. It is reassuring that the intensity ratio observed (Figure 6) for zero $\Delta T_{R}$ under helium CA conditions agreed well with that observed (Figure 4) for unimolecular fragmentations of reactant ions transmitted directly to the quadrupole collision cell, with no collision gas present in either the MIKES collision cell or the RF-only quadrupole. These two apparently very different sets of experimental conditions should both probe the same value of $\epsilon_{\mathrm{R}}^{\mathbf{0}}$.

A clearcut conclusion from comparison of Figures 4 and 6 is that the collisional energy loss $\Delta T_{R}(0-15 \mathrm{eV})$ was considerably larger than the internal energy exhibited by those ions that subsequently fragmented within the RF-only quadrupole cell (up to $5 \mathrm{eV}$, the maximum possible value corresponding to the unlikely circumstance that $f$ had its maximum value of unity in Figure 4). This conclusion is less firm than that drawn from the butylbenzene data obtained using the four-sector instrument, in view of the delay between energy selection and the time window for observation of the fragmentation. It is important to note that very similar results were obtained (not shown) for the Leu-enkephalin case when using helium pressures sufficient to attenuate the intensity by up to $40-50 \%$ instead of $15-20 \%$. This insensitivity to the intervention of multiple collision events probably implies that most ions subjected to several collisions were scattered out of the acceptance aperture of the electric sector. The impor-

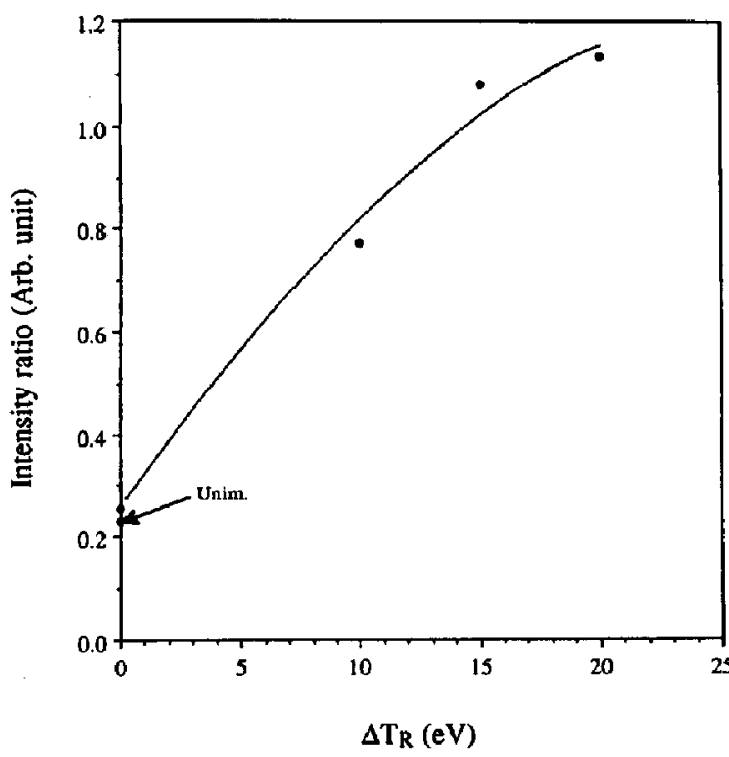

Figure 6. Intensity ratio of fragment ions ( $m / z 397$ and 425) from $(\mathrm{MH})^{+}$ions of Leu-enkephalin, collisionally activated (helium collision gas) at $8 \mathrm{keV}$ (laboratory reference frame) and selected for translational energy loss $\Delta \mathrm{T}_{R}$ but with no angular selection. The data were obtained using a $\mathrm{BEqQ}$ hybrid instrument (Figure 5). The point at $\Delta \mathrm{T}_{\mathbf{R}}=0$, marked "Unim," corresponds to that shown in Figure 4 for reactant ions subjected to no collisions at any point.

tance of this observation for the present work is that it makes unlikely the possibility that the observed effects reflect only the small fraction of ions subjected to more than one collision at $15-20 \%$ attenuation.

Theoretical calculations based upon the UggerudDerrick model [12] were performed for collisions of 8 $\mathrm{keV}$ protonated Leu-enkephalin with helium. The plots of calculated values for $\Delta T_{R}$ as a function of $\theta_{R}$ were qualitatively similar to those shown in Figure 3 . However, the values of $\Delta T_{R}$ at $\theta_{R}=0.29^{\circ}$ were $41 \mathrm{eV}$ and $33 \mathrm{eV}$, for $m_{a}=14 D_{a}$ and $100 D_{a}$, respectively. The corresponding values of $\left(Q / \Delta T_{R}\right)$ were 0.218 and 0.032 , and thus the maximum $Q$ values were $8.9 \mathrm{eV}$ and 1.1 $\mathrm{eV}$. (The exemplary value of $100 \mathrm{D}_{\mathrm{a}}$ for $\mathrm{m}_{\mathrm{a}}$ was chosen by analogy with previous findings [45] for low-energy CID of protonated Leu-enkephalin in an RF-only quadrupole collision cell. These findings could be reconciled in a self-consistent manner under the assumption [45] of a binary-collision model with an "impact portion" mass of $100 \mathrm{Da}$.) These theoretical predictions of maximum values for $\Delta T_{R}$ and $Q$, for the case of $\mathrm{m}_{\mathrm{a}}=14 \mathrm{D}_{\mathrm{a}}$, are too high by a factor of up to two, in comparison with the experimental results for which the maximum observable value of $\Delta \mathrm{T}_{\mathrm{R}}$ was about 25 $\mathrm{eV}$ (Figure 5) and the corresponding value of $\mathrm{Q}$ was 5 $\mathrm{eV}$ (assuming the maximum possible value of unity for the factor $f$ in Figure 4). However, larger values for $\mathrm{m}_{\mathrm{a}}$ bring the theoretical predictions into reasonable agreement with experiment. 


\section{Penta $\left(d_{3}\right)$ acetylgalactose}

This system was the subject of a preliminary study [15] that did not employ angular selection, but the available intensities of reactant ions were sufficient that a useful degree of angular collimation could be achieved in the present work. The reactant ions of interest $(\mathrm{m} / \mathrm{z}$ 343) are formed by expulsion of $\mathrm{CD}_{3} \mathrm{COOH}$ from protonated penta( $d_{3}$ )acetylgalastose (loss of the $d_{3}$-acetyl group from the anomeric carbon atom). It has been shown previously $[15,46,47]$ that the low-energy CID spectra of this reactant ion, in particular the intensity ratio for the fragment ions at $\mathrm{m} / \mathrm{z} 173$ and 172 , are highly sensitive to collision energy. For present purposes of an empirical index of internal energy content, only low-energy CID data obtained under essentially single-collision conditions are suitable. These results were published previously [15], but unfortunately the calibration curve of the intensity ratio versus $T_{\text {сом }}$ displayed a maximum, so that any observed intensity ratio can be interpreted in terms of either of two possible $T_{C O M}$ values. This ambiguity introduced considerable confusion into interpretation of the present data, particularly in comparisons of results obtained using the two tandem mass spectrometers. Accordingly, only a single example of the angle-resolved translational energy spectra, obtained using the hybrid instrument, will be shown and discussed (Figure 7).

The angle-resolved translational energy profiles of the collisionally activated survivor ions, observed at the energy resolving slit, were very similar to those obtained for $\mathrm{Cs}_{4} \mathrm{I}_{3}^{+}$ions (Figures 1 and 2 of ref 10 ). Figure 7 also shows examples of the delayed unimolecular fragmentations of reactant ions, selected for both $\Delta \mathrm{T}_{\mathrm{K}}$ and for $\theta_{\mathrm{K}}$, within the RF-only quadrupole. The fragment ion intensity ratio observed for reactant ions selected at $\theta_{\mathrm{R}}=0$ and $\Delta \mathrm{T}_{\mathrm{R}}=0$ (not shown) was the same as that shown for $\theta_{R}=0.18^{\circ}$ and $\Delta T_{R}=0$, although, of course, the signal level was much higher. Moreover, this same fragment intensity ratio of 0.25 was also observed for reactant ions transmitted to the $\mathrm{RF}$-only quadrupole cell in the absence of any CA whatsoever, and thus reflects $\epsilon_{\mathrm{R}}^{\circ}$ only. This is an impurtant consistency check, which indicates that the degree of angular collimation achieved was sufficient to reduce cross talk, between collisional scattering and energy dispersion to negligible proportions.

The maximum value observed for the ratio of intensities for $m / z 173$ and $m / z 172$ corresponded to a value of $T_{C O M}$ (or $Q_{\text {MAX }}$ ) of $4 \mathrm{eV}$ when transformed via the calibration curve published previously [15]. This value corresponds to the maximum in the calibration curve [15], referred to above, and is thus fortuitously not subject to the ambiguity in the assignment of the $T_{\text {Com }}\left(=Q_{\text {max }}\right)$ value. Of course, the actual mean value of $Q$ is probably appreciably smaller than this. How ever, since the corresponding value of $\Delta \mathrm{T}_{\mathrm{R}}$ was $25 \mathrm{eV}$, only a maximum of some $16 \%$ of $\Delta \mathrm{T}_{\mathrm{R}}$ can be accounted for by $\left(\epsilon_{R}-\epsilon_{R}^{0}\right)$. The larger values observed

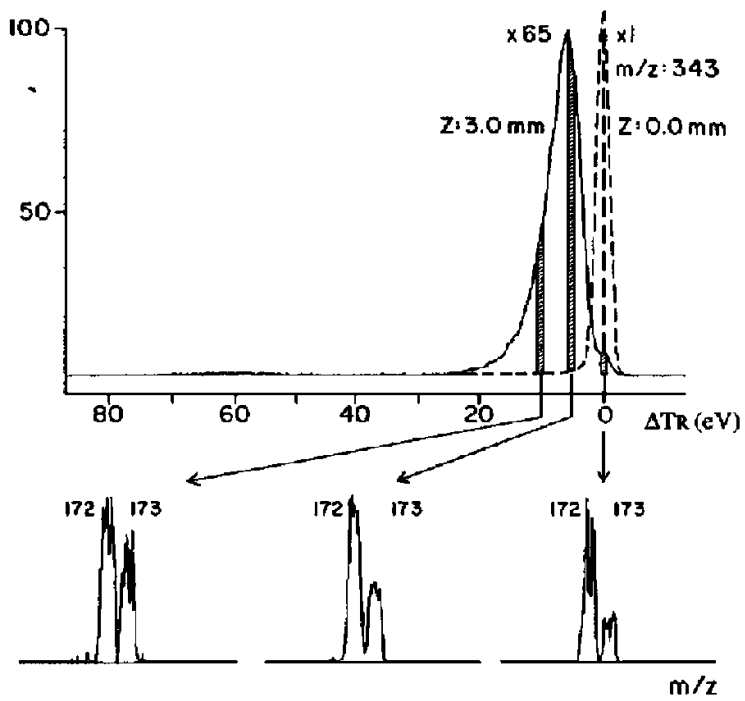

Figure 7. Delayed fragmentation spectra of ions of $m / z 343$ from penta $\left(d_{3}\right)$ acetylgalactose, collisionally activated at $8 \mathrm{keV}$ (helium collision gas) and selected for translational energy loss $\Delta T_{R}$. The reactant ion energy profile shown as a dashed curve was obtained with a 1-mm-high z-aperture centered on the main optical axis, corresponding to a scattering angle $\theta_{R}$ of $0.00 \pm$ $0.03^{\circ}$. The profile shown as a solid line was obtained using this z-aperture displaced by $3 \mathrm{~mm}$, corresponding to an angle of $0.18 \pm 0.03^{\circ}$. The partial fragment spectra were obtained by transmitting the reactant ions, selected at $\theta_{\mathrm{R}}=0.18 \pm 0.03^{\circ}$ and at values of $\Delta T_{R}$ of 0,5 , and $10 \mathrm{eV}$ (all $\pm 1 \mathrm{eV}$ ), to the $R F$-only quadrupole (indicated pressure $<10^{-8}$ mbar) of the $\mathrm{BEqQ}$ hybrid instrument.

for $\Delta T_{R}$ do exceed the lowest excitation energy of helium $(19.8 \mathrm{eV})$, so the possibility of target excitation cannot be ruled out in these cases. However, the same general conclusion holds for cases (including some illustrated in Figure 7) for which $\Delta \mathrm{T}_{\mathrm{R}}$ is much smaller then $19.8 \mathrm{eV}$.

\section{Conclusions}

The principal conclusion of the present work is that, in $\mathrm{CA}$ at kiloelectronvolt energies, a major sink for the translational energy loss $\Delta T_{R}$ of the reactant ion is the recoil energy of the target. Unfortunately, it was not possible to establish this conclusion for ions that are large in the sense of Derrick and co-workers [3-6]. For low-mass targets such as helium, at larger scattering angles $\theta_{R}$, this recoil energy accounts for most of $\Delta T_{R}$. This conclusion is based upon two features of the present work, that is, the experimental exclusion of internal energy of the target as a possible sink for $\Delta T_{R}$ via use of inert gas targets and the independent estimation of internal energy deposition in the reactant ion. Although the measurement of internal energy could not be achieved in an absolute sense for two of 
the reactant ions investigated, the empirical indices of internal energy were defined so that they provided upper bounds to the true mean value in each case. In addition, this conclusion is independent of the simple collision theories $[11,12]$ used here and also in the companion article [10]. These theoretical considerations are, however, consistent with the conclusions based on the experimental results.

The large values of $\Delta \mathrm{T}_{\mathrm{R}}$ and broad asymmetric peaks, observed for helium as a target in previous observations [3-9] of MIKES peaks for fragment ions, reflect the same phenomenon. The range of $\Delta T_{R}$ values corresponding to the instrument-defined range for $\theta_{\mathrm{R}}$ is much larger in the case of a low-mass target, so that integration over $\theta_{\mathrm{R}}$ implies superposition of $\mathrm{R}^{+}$survivor ions with a wider range of $\Delta T_{R}$ values in the case of helium than for heavy targets. Because nothing is known about the $R^{+}-G$ interaction potential, no predictions can be made [11] concerning the relative probabilities for the various $\left(\Delta T_{R}, \theta_{R}\right)$ combinations, and thus no quantitative interpretations of the peak shapes are possible. In this context, it is appropriate to point out that the magnitude of the discrepancy between $Q$ and $\Delta \mathrm{T}_{\mathrm{R}}$ increases with $\theta_{\mathrm{R}}$ (and thus with $\Delta \mathrm{T}_{R}$ ), and is quite small at lower values of $\theta_{R}$ (see Figure 3 ). The problem addressed in the present work is therefore serious only for the larger values of $\theta_{R}$ (and thus of both $\Delta T_{R}$ and $Q$ ). However, it is precisely these more violent collisions that are the most effective in promoting $\mathrm{CID}$, so the problem remains important in a practical sense.

An important assumption underlying these conclusions concerns the role of radiative cooling of the activated reactant ions. For this effect to account for the present observations would require emission of several electronvolts with high efficiency within a few microseconds. As discussed in the companion article [10], UV-visible emission probabilities cannot compete with internal conversion to the ground electronic state, while infrared radiative cooling of vibrationally excited ions is much too slow, as confirmed recently by Uechi and Dunbar [48] for the case of the butylbenzene molecular ion.

Although the present work is clearly not the last word on CID of large ions at kiloelectronvolt energies, it does provide some insight into the $C A$ step. The conclusion that the energy shifts $\Delta T_{R}$ are mainly a consequence of the physics of the collisions, rather than of the much more complex phenomenon of kinetic shifts for chemical fragmentation, offers some hope that the technical problem [49] of correcting the linked-scan locus of the fragment ion analyzer $\left(\mathrm{E}_{2} \mathrm{~B}_{2}\right)$ of the four-sector instrument to account for $\Delta T_{R}$ might be susceptible to a reasoned solution.

\section{Acknowledgment}

The authors are indebted to J. L. C. Wright (National Research Council) for the sample of penta-d ${ }_{3}$-acetylgalactose.

\section{References}

- 1. Durup, J. In: Recent Developments in Mass Spectrometry; Ogata, K.; Hayakawa, T., Eds.; University of Tokyo Press: Tokyo, 1970; pp 921-934.

2. Los, J.; Govers, T. R. In: Collision Spectroscupy; Couks, R. G., Ed.; Plenum: New York, 1978; pp 289-356.

3. Neumann, G. M; Derrick, P. J. Org. Mass Spectrom. 1984, 19, $165-170$.

4. Neumann, G. M.; Sheil, M. M.; Derrick, P. J. Z. Naturforsch. 1984, 39a, 584-592.

5. Sheil, M. M.; Derrick, P. J. Org. Mass Spectrom. 1988, 23, $429-435$.

6. Uggerud, E.; Derrick, P. J. Z. Naturforsch. 1989, 44a, 245-246.

7. Bricker, D. L.; Russell, D. H. J. Am. Chem. Soc. 1986, 108, 6174-6179.

8. Guevremont, R; Boyd, R. K. Rapid Commun. Mass Spectrom. 1988, 2, 1-5.

9. Alexander, A. J.; Thibault, P.; Boyd, R. K. I. Am. Chem. Soc. 1990, 112, 2484-2491.

10. Thibault, P.; Alexander A. J.; Boyd, R. K. J. Am Soc. Mass Spectrom. 1993, 4, 835-844.

11. Boyd, R. K.; Kingston, E. E.; Brenton, A. G; Beynon, J. H. Prac. Roy. Soc. Lond. 1984, A392, 59-88, 89-106.

12. Uggerud, E.; Derrick, P. J. J. Phys. Chem. 1991, 95, 1430-1436; 1993, 97, 5443-5444.

13. Cerny, R. L.; Tomer, K. B; Giblin, D. E. Procedings of the 34th ASMS Conference on Mass Spectrometry and Allied Topics; Cincinnati, OH, 1986; pp 99-100.

14. Gross, M. L.; Tomer, K. B.; Cerny, R. L.; Giblin, D. E. In: Mass Spectrometry in the Analysis of Large Molecules; McNeal, C. J., Ed; Wiley: New York, 1986; pp 171-190.

15. Alexander, A. J.; Thibault, P. Rapid Commun. Mass Spectrom. 1988, 2, 224-228.

16. Alexander, A. J.; Boyd, R. K; Thibault, P; Tomer, K. B. Proceedings of the 37th ASMS Conference on Mass Spectrometry and Allied Topics; Miami Beach, FL, 1989; PF 226-227.

17. Ballard, K, D.; Gaskell, S. J. J. Am. Soc. Mass Spectrom. 1992, 3, 644-655.

18. Proctor, C. J.; Brenton, A. G.; Beynon, J. H.; Kralj, B.; Marsel, J. Int. J. Mass Spectrom. Ion Phys. 1980, 35, 393-403.

19. Proctor, C. J.; Kralj, B.; Brenton, A. G.; Beynon, J. H. Org. Mass Spectrom. 1980, 15, 619-631.

20. Griffiths, I. W.; Mukhtar, E. S.; March, R. E.; Harris, F. M.; Beynon, J. H. Int. J. Mass Spectrom. Ion Phys. 1981, 39, 125-132.

21. Griffiths, I. W.; Mukhtar, E. S.; Harris, F. M.; Beynon, J. H. Int. I. Mass Spectron. Ion Phys. 1982, 43, 283-292.

22. Harrison, A. G.; Lin, M. S. Int. J. Mass Spectrom. Ion Phys. 1983, 51, 353-356.

23. Chen, J. H.; Hays, J. D; Dunbar, R. C. J. Phys. Chem. 1984, 88, 4759-4764.

24. Boyd, R. K.; Harris, F. M.; Beynon. J. H. Int. J. Mass Spectrom Ion Processes 1985, 66, 185--194.

25. Baer, T.; Drutuit, O.; Mestdagh, H.; Rolando, C. 1. Phys, Chem 1988, $92,5674-5679$.

26. Brown, P. Org. Mass Spectrom. 1970, 3, $1175-1186$.

27. Franchetti, V.; Carmody, J. J.; Krause, D. A.; Cooks, R. G. Int I. Mass Spectrom. Ion Phys. 1978, 26, 353-358.

28. Laramee, J. A.; Carmody, J. J.; Cooks, R. G. Int. J. Mass Spectram. Ion Phys. 1979, 31, 333-343.

29. Fedor, D. M.; Cooks, R. G. Anal. Chem. 1980; 52, 679682.

30. Laramee, J. A.; Hemberger, P. H.; Cooks, R. G. Int. J. Mass Spectrom. Ion Phys. 1980, 33, 231-241.

31. Hubik, A. R.; Hemberger, P. H.; Laramee, J. A.; Cooks, R. G. J. Am. Chem. Soc. 1980, 102, 3997-4000.

32. Hemberger, P. H.; Laramee, J. A.; Hubik, A. R.; Cooks, R. G. J. Phys. Chem. 1981, 85, 2335-2340. 
33. Verma, S.; Ciupek, J. D.; Cooks, R. G.; Schoen, A. E.; Dobberstein, P. Int. J. Mass Spectrom. Ion Phys, 1983, 52, 311-318.

34. Mason, R. S.; Farncombe, M. J.; Jenrings, K. R.; Cooks, R. G. Int. J. Mass Spectrom. Ion Phys. 1982, 42, 327-330.

35. McLuckey, S. A.; Verma, S.; Cooks, R. G.; Farncombe, M. J.; Mason, R. S.; Jennings, K. R. Int. J. Mass Spectrom. Ion Phys. 1983, 48, 423-426.

36. Todd, P. J.; Warmack, R. J.; McBay, E. J. Int. J. Mass Spectrom. Ion Phys. 1983, 50, 299-313.

37. Waddell, D. S.; Buyd, R. K.; Brenton, A. G.; Beynur, J. H. Int. J. Mass Spectrom. Ion Phys. 1986, 68, 71-90.

38. de Bruijn, D. P.; Los, I. Rev. Sci. Instrum. 1982, 53, 1020-1026.

39. van der Zande. W. J.; de Bruijn, D. P ; Los, J.; Kistemaker, P. G. Int. J. Mass Spectrom. Ion Processes 1985, 67, 161-170.

40. Harrison, A. G.; Mercer, R. S.; Reiner, E. J.; Young, A. B.; Boyd, R, K,; March, R. E.; Porter, C. J. Int. J. Mass Spectrom. Ion Processes 1986, 74, 13-31.
41. Singh, S.; Harris, F. M.; Boyd, R. K.; Beynon, J. H. Int. J. Mass Spectrom. Ion Processes 1985, 66, 131-149, 151-166.

42. DeKrey, M. J.; Kenttamaa, H. I.; Wysocki, V. H.; Cooke, R. G. Org. Mass Spectrom. 1986, 21, 193-195.

43. Vincenti, M.; Horning, S. R.; Cooks, R. G. Org. Mass Spectrom. 1988, 23, 585-593.

44. Cooks, R. G.; Ast, T.; Kralj, B.; Kramer, V,; Zigon, D. J. Am. Soc. Mass Spectrom. 1990, 1, 16-27.

45. Alexander, A. J.; Boyd, R. K. Int. I. Mass Spectrom. Ion Processes 1989, 90, 211-240.

46. Mueller, D. R.; Domon, B. M.; Blum, W.; Raschdorf, F; Richter, W. Biomed. Enviran. Mass Spectrom. 1988, 15, 441-446.

47. Guevremont, R.; Wright, J. L. C. Rapid Commun. Mass Spectrom. 1988, 2, 47-49.

48. Uechi, G. T.; Dunbar, R. C. J. Chem. Phys. 1992, 96, 8897-8905. 49. Boyd, R. K. Int. T. Mass Spectrom. Ion Processes 1987, 75, 243-264. 